\title{
Genital Prolapse at the End Term of Pregnancy: A Case Report
}

\author{
Afaf Abdalla* \\ Department of Obstetrics and Gynecology Nursing, Alneelain University, Sudan
}

*Corresponding author: Afaf Abdalla, Department of Obstetrics and Gynecology Nursing, Alneelain University, Sudan, Tel: 00249912187802; Email: aabdalla642@gmail.com

Rec date: May 31, 2017; Acc date: Aug 25, 2017; Pub date: Aug 30, 2017

Citation: Abdalla A (2017) Genital Prolapse at the End Term of Pregnancy: A Case Report. Gynecol Obstet Case Rep Vol.3:No.2:54.

\section{Abstract}

Uterine prolapsed is a rare condition occurs during pregnancy and lead to many complications. Usually conservative treatment approached to use a vaginal pessary accompanied with bed rest. This current case exhibited obvious role of a vaginal pessary in restoring the prolapsed organ till the time of labor and may expect delivery at home. When studying the style of labor, Gynecologist should bear in their mind the cervical irritation and edema. Along with the genital prolapsed which may affect normal vaginal delivery.

Keywords: Uterine prolapse; Third trimester; Pregnancy; Vaginal pessary

\section{Introduction}

Uterine prolapse is a course of genital prolapsed, which happens when the uterus slips from its place into the vagina. Although the incidence rate of uterine prolapse has declined during the past decade in developed nations due to the gradual decrease in parity, approximately $50 \%$ of all adults who have delivered a near-term infant have some degree of clinically apparent genital prolapsed [1].

Genital prolapse is a condition that can dramatically affect quality of life causing specific problems like inability to walk, sitting, lifting and bending [2].

Genital prolapse may start before labor. Nevertheless, in the most of cases, pregnancy has advertised effect on a preexisting prolapse. The concomitant phenomenon of a third trimester pregnancy with a significant level of pelvic organ prolapse is extremely rare [3].

Settlement of the prolapse in later weeks of pregnancy is expected as there is an increase in the uterine volume, which helps to posture on the pelvic brim. Now we discuss a case of a woman who presented with genital prolapsed before to deliver her child.

\section{Case Report}

A 28-year-old lady pregnant four times, Para 3, living 2 presented at 36 weeks of gestation, she complains with from low backache, abdominal pain and a mass in her vagina for 3 days. In her current pregnancy was complicated from the beginning and second trimesters. She found a mass per vagina, which appears suddenly. She had no history of chronic constipation, lifting heavy objects or chronic cough. When we do a physical examination of the reproductive tract system, observed that the lower part of the uterus gets out. The cervix of the uterus was bulging and appear after the vaginal opening, but easily to return back to it is the normal position. The cervical OS was closed and other systems were normal. Ultrasound shows normal cervical anatomy. Therefore, she was admitted to the hospital and the protruding part was returning back without surgical intervention. She was given antibiotics, intravenous Betamethasone $(12 \mathrm{mg}$, two times with 12 hours apart) and to continue on prophylactic tocolytic, per oral every 8 hours daily for one week and was counseled about importance of bed rest at home. After four weeks, she had an uneventful normal vaginal delivery at home and the protruding part return without intervention. She came back on the 25th day after delivery with the appearance of the mass for the second time. On testing the lower uterine part was outside the vaginal opening and the uterus was of appropriate measurement. We did not find any puerperal infection elsewhere. The patient was moved over a regime of antibiotic tabs, as well as iron and advised for Sling operation plus tubal ligation after puerperium.

\section{Discussion}

Protrusion of the uterus not more occur during gestation nevertheless happened more common in non-pregnant elderly women. It may develop for the first time during gestation, but in the most of condition, pregnancy could consider a direct cause of prolapsed uterus. When more or less stage of protrusion is found prior gestation, commonly continues till the pregnancy advance to a stage where spontaneous reducible occurs. In many conditions, a symptom of the prolapsed happens in the last trimester, if it takes place at the beginning of the pregnancy, can resolve after labor and delivery spontaneously. 
Genital prolapsed during pregnancy may show with a broad scope of clinical features, pelvic heaviness, lower back ache, urinary tract symptoms (unable to pass urine, uncontrol to pass urine), lower uterine part inflammations and ulcerations cervical mucosal. Consistently, side effects reported also range from mild discomfort, cervical irritation, urinary tract infection, acute urinary retention, abortion, and even women mortality $[4,5]$.

In that respect are several risk factors for uterine prolapsed including, parity, advancing age, smoking, obesity hysterectomy, some connective tissue disorders, congenital abnormalities, positive family history of uterine prolapsed and any chronic conditions that accompanied by a repetitive increase in intra-abdominal pressure such as chronic constipation. Parity contained several components (pregnancy, labor, delivery) that each of them may lead to pelvic relaxation. On that point is an argument about the effect of labor, without vaginal delivery; most of studies suggest that it has a negligible effect on the uterine prolapsed but some data have yielded opposite results [6,7].

Most problem occur, along a genital prolapsed during gestation are delivery before term and fetal demise which including miscarriage, urinary tract infection, beside urinary retention. However, women death has been observed as a complication of genital prolapsed during pregnancy.

Thus, some gynecologist advised for elective cesarean section as a proper way of delivery to avoid suspected complication during labor. Even then, like that condition, if the woman already possesses a favorable soft cervix. A gynecologist does not perform a cesarean section. The conservative management is preferable choice for management during pregnancy because uterine prolapsed usually resolves without intervention after delivery. Conservative management consists of genital hygiene and bed rest in a slight Trendelenburg position, which is managed with close follow-up on an outpatient basis or hospitalization [8].

The condition of this is consistent with finding from a study conducted by Eddib et al. from the USA, reported a case of successful pregnancy in a 44-year-old patient with pre-existing total genital prolapsed (procedentia), came at the first trimester with unplanned pregnancy, prolapse persisted up to the end of the second trimester, choice for her conservative management, along with bed rest and local treatment of the desiccated cervix with emollients. She had an uneventful vaginal delivery and later treatment complete with removal of the uterus through the vagina (vaginal hysterectomy) with the anterior, posterior repair and sacrospinal ligament fixation after few months [9].

A vaginal pessary is a short period treatment to alleviate more worsen of the condition. On the other hand, in that respect an urgent need to search about the successes of pessaries and a kegal exercise in developing countries to take decisions towards national strategies and guidelines that demonstrate support and steering with a realistic and applicable monitoring system [10] (Figure 1).

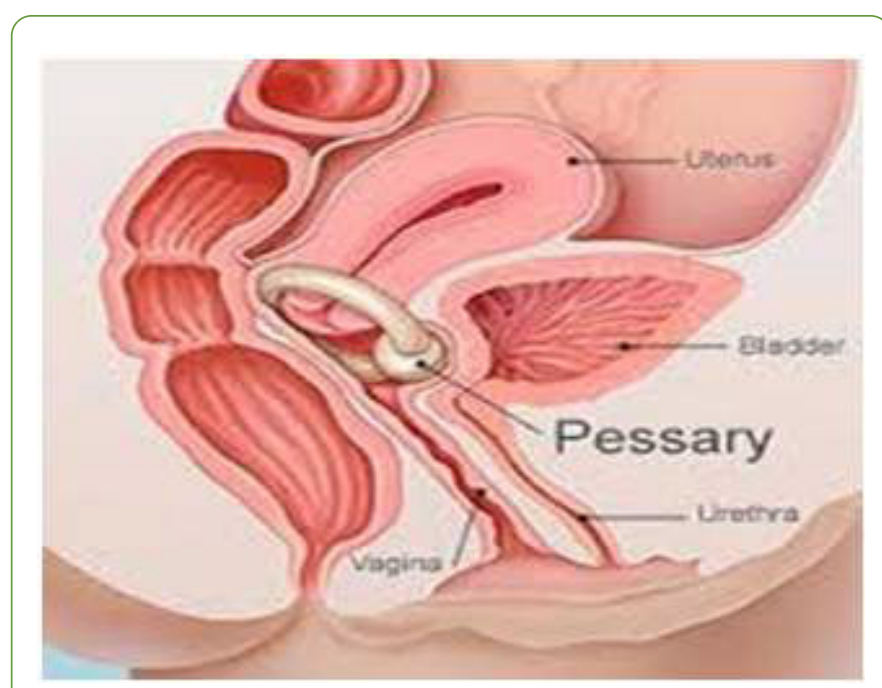

Figure 1 Vaginal pessary.

Most of the women sat in labor and deliver normally, however, affected women may be in danger of cervical dystocia during labor that may call for intervention during labor achieve uneventful gestation. When studying the style of delivery, spontaneous delivery or a planned LSCS, a gynecologist should observe for cervical irritation and edema which may guide to complicate the vaginal delivery [11].

\section{Conclusion}

Genital prolapse concurrent with pregnancy is a seldom condition occurring during pregnancy. Early identification of this circumstance is crucial and, together with proper management, enables avoidance of the related complications, such as preterm labor and trauma during delivery. Conservative management of these women during pregnancy can result in an uncomplicated, spontaneous delivery. Basically, the degree of success depending on the severity of the prolapse.

\section{Acknowledgements}

We are very grateful to the patient for co-operation in completing this small piece of work.

\section{References}

1. Brown HL (1997) Cervical prolapse complicating pregnancy. J Natl Med Assoc 89: 346-348.

2. Hardee K, Gay J, Blanc AK (2012) Maternal morbidity: Neglected dimension of safe motherhood in the developing world. Glob Public Health 7: 603-617.

3. Meydanli MM, Ustun Y, Yalcil OT (2006) Pelvic organ prolapse complicating third trimester pregnancy. Gynecol Obstet Invest 61: 133-134.

4. Tinelli A, Malvasi A, Rahimi S, Negro R, Vergara D, et al. (2010) Age-related pelvic floor modifications and prolapse risk factors in postmenopausal women. Menopause 17: 204-212. 
5. Daskalakis G, Lymberopoulos E, Anastasakis E, Kalmantis K, Athanasaki A, et al. (2007) Uterine prolapse complicating pregnancy. Arch GynecolObstet 276: 391-392.

6. Mirza-Moradi M, Zahra Heidar, Saleh M (2015) Uterine prolapse during pregnancy: A case report. Novel Biomed 3: 222-224.

7. Kim JO, Jang SA, Lee JY, Yun NR, Lee SH, et al. (2016) Uterine prolapse in primigravid woman. Obstet Gynecol Sci 59: 241-244

8. Kart C, Aran T, Guven S (2011) Stage IV C prolapse in pregnancy. Int J Gynaecol Obstet 112: 142-143.
9. Eddib A, Allaf MB, Lele A (2010) Pregnancy in women with uterine 12. procedentia: A case report. J Reprod Med 55: 67-70.

10. Radl CM, Rajwar R, Aro AR (2012) Uterine prolapse prevention in Eastern Nepal: The perspectives of women and health care professionals. Int J Womens Health 4: 373-382.

11. Sunita SP, Sreevani K, Vrunda Chaudhary, Rajesh Kaul (2016) Acute uterine prolapse. JBCR 3: 28-30. 